\title{
Metabolomics changes in brain-gut axis after unpredictable chronic mild stress
}

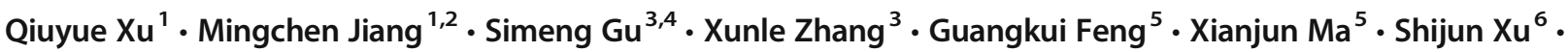 \\ Erxi $\mathrm{Wu}^{7,8}$ • Jason $\mathrm{H} \mathrm{Huang}^{7,8} \cdot$ Fushun Wang ${ }^{3}$ (D)
}

Received: 1 March 2021 / Accepted: 2 August 2021 / Published online: 8 February 2022

(C) The Author(s) 2021

\begin{abstract}
Background Major depressive disorder is a leading cause of disability worldwide, affecting up to $17 \%$ of the general population. The neural mechanisms of depression, however, are yet to be uncovered. Recently, attention has been drawn to the effects of dysfunctional brain-gut axis on depression, and many substances have been suggested to be involved in the communication between the gut and brain, such as ghrelin.

Methods We herein systematically examined the changes of metabolomics after unpredictable chronic mild stress (UCMS)induced depression-like behaviors in rats and compared the altered metabolites in the hippocampus and jejunum samples.

Results Our results show that many metabolites significantly changed with UCMS both in the hippocampus and jejunum, such as L-glutamine, L-tyrosine, hydroxylamine, and 3-phosphoglyceric acid. Further studies suggested that these changes are the reasons for anxiety-like behaviors and depression-like behaviors in UCMS rats and also are the reasons for hippocampal neural plasticity.

Conclusions Coexistence of brain and gut metabolic changes in UCMS-induced depressive behavior in rats suggests a possible role of brain-gut axis in depression. This study provides insights into the neurobiology of depression.
\end{abstract}

Keywords Major depressive disorders · UCMS · MDD · Brain-gut axis · Metabolomics

\section{Introduction}

Major depression is affecting more than $17 \%$ of populations worldwide (Otte et al. 2016; Putnam et al. 2017), and the World Health Organization anticipated that depression will become the first health problem in 2020 (Gu, et al. 2020). Despite recent research advances, fewer than half of major

Qiuyue $\mathrm{Xu}$, Mingchen Jiang and Simeng Gu contributed equally to this work.

Guangkui Feng

lygfgk@163.com

Fushun Wang

13814541138@163.com

1 School of Nursing, Nanjing University of Chinese Medicine, Nanjing 210023, China

2 Department of Pediatrics, Hospital of Nanjing University of Chinese Medicine, Nanjing 210004, China

3 Institute of Brain and Psychological Science, Sichuan Normal University, Chengdu 610066, China depressive disorder (MDD) patients respond to antidepressant medications, thus highlighting the need to characterize the specific pathophysiology of MDD. However, the majority of previous studies have focused on the brain-specific mechanism and circuits, with only limited focus on the potential contributions of peripheral organs (Qin et al. 2019). Recently, attention has been drawn to the brain-gut axis, and

4 Department of Psychology, Jiangsu University Medical School, Zhenjiang 212013, China

5 Department of Neurology, Lianyungang Hospital of Chinese Medicine, Nanjing University of Chinese Medicine, Lianyungang 222000, China

6 School of Pharmacy, Chengdu University of Traditional Chinese Medicine, Chengdu 611137, Sichuan, China

7 Department of Neurosurgery, Baylor Scott \& White Health, Temple, TX 76508, USA

8 Department of Surgery, Texas A\&M University College of Medicine, Temple, TX 76508, USA 
the association between gut-brain axis with depression has been recognized. The brain-gut axis is a bidirectional, multifaceted communication system (Cryan et al. 2019), and the gut and brain might affect each other via the enteric nervous system, the parasympathetic system, the immune system, and microbial metabolites (Duman et al. 2016). For example, the vagus nerve represents the main component of the parasympathetic nervous system and plays an important role in depression (Breit et al. 2018). Meanwhile, brain activities can also be regulated by brain-gut peptides, such as ghrelin and insulin that are released by the gut (Sharon et al. 2019).

More recently, the microbiota has emerged as a key player in control of the brain-gut axis, especially during conditions of stress provoked by real or perceived homeostatic challenge. The gut microbiota, microorganisms that reside in the gastrointestinal (GI) tract, can modulate the activities of the central nervous system. For example, it is found that transplantation of microbiota from depressed patients to germ-free rats led to depressive behaviors (Zheng et al. 2016). In addition, it is found that microbiota-based interventions can alter the monoamine neurotransmitters in the brain (Strandwitz 2018), including norepinephrine (NE), dopamine (DA), serotonin, gamma-aminobutyric acid, and glutamate and metabolites (Baj et al. 2019; Xu et al. 2020). Despite increasing evidence associating affective disorders with imbalanced brain-gut interactions, the molecular mechanism of the brain-gut axis has yet to be elucidated in neuropsychiatric disorders such as depression. We herein investigated the metabolic molecule changes in the brain and intestine using gas chromatography-mass spectrometry (GC-MS/MS) in unpredictable chronic mild stress (UCMS) rat model of depression. As one of the most advanced analytic methods, GC-MS/MS combines gas chromatography and mass spectrometry to perform effective qualitative and quantitative analysis within a biological specimen. In this study, we used GC-MS to investigate the metabolites, especially those associated with the neurotransmitter metabolism in the hippocampus and intestines, and found many metabolites have significant change in both the brain and the intestines after introduction of UCMS to rats. These metabolic alterations are closely associated with neuroplasticity changes in the hippocampus, which could be implicated in the pathogenesis of depression.

\section{Materials and methods}

\section{Animals and reagents}

Depression occurrence in female patients is twice as many as male patients, because they are susceptible to hormone changes during the menstrual circle. This might the reason that a majority of stress models are using male rodents instead of females (Gu et al. 2018a). In this study all male Sprague-
Dawley (SD) rats (weighing between 200 and 220 g, age, 68 weeks) were purchased from Nanjing Qing Longshan experimental animal company (Nanjing, China). Rats were kept under standard conditions. The animals were placed under a 12/12-h light/dark cycle ( $7 \mathrm{am} / 7 \mathrm{pm})$ and the prescribed temperature conditions $\left(22 \pm 2{ }^{\circ} \mathrm{C}\right)$. Food and water were provided to be accessed freely. All animal experiments are conducted in accordance with Guide for the Care and Use of Laboratory Animals (the National Research Council of the National Academies, Eighth Edition, 2011 revision) and carried out in accordance with the NIH Guide for the Care and Use of Laboratory Animals (NIH Publication No. 85-23, 1985, revised 1996) and also performed strictly under the institutional guidelines of the Animal Care and Use Committee of Nanjing University of Chinese Medicine (Nanjing, China).

Methoxyamine hydrochloride, pyridine, N,OBis(trimethylsilyl)trifluoroacetamide (BSTFA) with $1 \%$ trimethylchlorosilane (TMCS), fluoxetine, and $1,2{ }^{13} \mathrm{C}_{2}-$ myristic acid were purchased from Sigma-Aldrich (St. Louis, MO, USA). N-hexane was purchased from ROE Scientific (St. Louis, MO, USA). Methanol of MS grade was supplied by Merck Millipore (Billerica, MA, USA). Ultrahigh-purity water was prepared by Millipore-Q system (Millipore Corporation, Billerica, MA, USA).

\section{Unpredictable chronic mild stress (UCMS) procedure}

Iqbal et al compared subchronic variable stress (SCVS) and unpredictable chronic mild stress (UCMS) models to evaluate the susceptibility versus resilient phenotypes in male and female rats. SCVS induced depression-like behaviors in female rats only. The UCMS paradigm was more likely to induce depression-like behaviors in male rats (Iqbal et al. 2020). Based on the above, we replicated the UCMS model and involved experiments. Thirty-six Sprague-Dawley rats (SD rats) were randomly divided into three groups: group I, control group (control+0.9 $\%$ saline); group II, UCMS model of depression group (UCMS+0.9\% saline); and group III UCMS with fluoxetine (UCMS + fluoxetine $2.4 \mathrm{mg} \cdot \mathrm{kg}^{-1}$ ). Group III started from the third week and were given continuous intragastric drug administration for 3 weeks continuously, 3 times a day. Fluoxetine solution was dissolved in $0.9 \%$ saline ( $\mathrm{Gu}$ et al. 2018b). Rats in group II and group III were exposed to consecutive UCMS for 5 weeks, while rats in group I were exempt. In summary, rats were exposed to the following stressors for each of the 24-h period: wet bedding $(24 \mathrm{~h})$, tail pinch $(5 \mathrm{~min})$, swimming in cold water $\left(4{ }^{\circ} \mathrm{C}, 5 \mathrm{~min}\right)$, restraint $(4 \mathrm{~h})$, food or water deprivation $(24 \mathrm{~h})$, light/dark cycle inversion (24 h), cage tilting (4 h), and swimming in hot water $\left(42{ }^{\circ} \mathrm{C}, 5 \mathrm{~min}\right)$. 


\section{Behavioral tests and serum corticosterone test}

After the UCMS procedure, the rats were tested with sucrose preference test (SPT) which was performed on day 35, open field test (OFT) on day 36, and forced swim test (FST) on day 37, as we previously reported ( $\mathrm{Gu}$ et al. 2018a). All behavior tests were performed between 9:00 am and 4:00 pm by an observer who was blinded to the treatment groups ( $\mathrm{Gu}$ et al. 2018b). The FST experiments were carried out between 9:00 am and 12:00 pm, in a plastic bucket $(50 \mathrm{~cm}$ high and $25 \mathrm{~cm}$ diameter) containing $30 \mathrm{~cm}$ of water at a temperature of around $25^{\circ} \mathrm{C}$. The rats of each group were individually placed in the bucket for $5 \mathrm{~min}$. The experiments were recorded with the Depression Scan software, which automatically analyzed the immobile time of each group of rats. The rats were blowdried and put back into the cage after each experiment. The open field test is a depression-like behavior test that lasted for $5 \mathrm{~min}$. The rats were placed in the center square of an open filed box $(60 \times 60 \times 40 \mathrm{~cm})$ which was divided into nine squares. The parameters used for analysis included the total travel distance, time spent in the central area (immobility time), and the grooming times. The sucrose preference test is a reward-based test to examine anhedonia. The rats were fastened for $20 \mathrm{~h}$ and then provided with two bottles, with one containing $2.5 \%$ sucrose solution and the other containing pure water. The recording lasted for $2 \mathrm{~h}$. The percentage preference was calculated according to the following formula: $\%$ preference $=$ sucrose intake $/($ sucrose intake + water intake $) \times$ $100 \%$. Blood samples were obtained from the tail in the morning every Saturday to test plasma sample with enzyme immunoassay (EIA) kits.

\section{Hippocampus pretreatment for metabolomics analysis}

Sample preparation for GC-MS analysis was revised according to previous reports (Qian et al. 2019). Briefly, $20 \mathrm{mg}$ of frozen hippocampus was homogenized in $500 \mu \mathrm{L}$ of $\mathrm{MeOH}$, and $20 \mu \mathrm{L}$ of ice methanol containing $1,2-{ }^{13} \mathrm{C}_{2}$-myristic acid was added to a final concentration of $10 \mu \mathrm{g} / \mathrm{mL}$ and vortexed for $5 \mathrm{~min}$. The mixture was centrifuged at $27400 \times g$ for 10 min, and $300 \mu \mathrm{L}$ of the mixture supernatant was dried at $45{ }^{\circ} \mathrm{C}$ and $15 \mathrm{kPa}$ to evaporate $\mathrm{MeOH}$. After drying, the sample was added to $50 \mu \mathrm{L}$ of $15-\mathrm{mg} / \mathrm{mL}$ methoxyamine pyridine and vortexed for $5 \mathrm{~min}$. Then, it was vortexed for $3 \mathrm{~min}$ and shaken at $30{ }^{\circ} \mathrm{C}$ for 90 min with the ThermoMixer C (Eppendorf, Hamburg, Germany). Immediately, thereafter, $50 \mu \mathrm{L}$ of BSTFA trifluoroacetamide (BSTFA) containing $1 \%$ TMS was added, and the sample was shaken again at $37{ }^{\circ} \mathrm{C}$ for $0.5 \mathrm{~h}$. The mixture was then transferred to a sampler vial and subjected to GC-MS analysis.
Jejunum pretreatment for metabolomics analysis

We chose jejunum to represent gut samples for analysis according to previous reports. The jejunum begins with the duodenal jejunum curve, especially the upper third is the most abundant part of the folds, the most important part of nutrient absorption, and the most important part of blood supply. Changes in jejunum mRNA expression can trigger neuromodulation and endocrine-related pathways and play a key role in metabolic-related diseases (Liang et al. 2018). Similarly, researchers are attempting to harness the advantages of the gut-brain axis to prevent neurocognitive disorders by enhancing intestinal health; the conclusion was drawn by focusing on the relevant indicators of jejunum samples (Wang et al. 2020). A 50-mg jejunum was added to $1-\mathrm{mL} \mathrm{MeOH}$ and grounded for $15 \mathrm{~min}$; afterward, $20 \mu \mathrm{L}$ of methanol (containing $12.5 \mu \mathrm{g} / \mathrm{mL} 1,2-{ }^{13} \mathrm{C}_{2}$-myristic acid) was added for vortexing for $3 \mathrm{~min}$ to precipitate the protein and mix the internal standard with the sample. The mixture was centrifuged at $27400 \times g$ for $10 \mathrm{~min}$ at $4{ }^{\circ} \mathrm{C}$, and $300 \mu \mathrm{L}$ of 300 supernatants was dried at $45{ }^{\circ} \mathrm{C}$ and $15 \mathrm{kPa}$ to evaporate $\mathrm{MeOH}$. After drying, the sample was added to $50 \mu \mathrm{L}$ of a concentration of $15-\mathrm{mg} / \mathrm{mL}$ methoxyamine pyridine and vortexed for $5 \mathrm{~min}$, and then the sample was reconstituted by shaking at $30^{\circ} \mathrm{C}$ for $90 \mathrm{~min}$ with the ThermoMixer $\mathrm{C}$ (Eppendorf, Hamburg, Germany). Immediately after, $60 \mu \mathrm{L}$ of BSTFA trifluoroacetamide containing 1\% TMS was added, vortexed for $1 \mathrm{~min}$, and shaken again at $37{ }^{\circ} \mathrm{C}$ for $0.5 \mathrm{~h}$. Subsequently, $60 \mu \mathrm{L}$ of the supernatant was centrifuged at $45300 \times g$ for $10 \mathrm{~min}$ and then injected into the GC-MS for analysis. All derivatization must be carried out in an anhydrous environment. Quality control (QC) samples of the hippocampus and jejunum were also prepared and derivatized according to the abovementioned protocol and were run after every 12 samples to monitor the retention time and elution order of the metabolites.

\section{GC-MS conditions for metabolomics analysis}

Metabolomics analysis of hippocampus and jejunum samples was performed using a Thermo Trace 1310-TSQ 8000 gas chromatograph system coupled to a mass spectrometer. Each $1 \mu \mathrm{L}$ of the derivative sample was injected onto a TG-5MS capillary column $(0.25 \mathrm{~mm} \times 30 \mathrm{~m} \times 0.25 \mu \mathrm{m}$, Thermo Fisher, San Jose, CA, USA), and the excitation mode was separated by a ratio of $20: 1$. The gradient heating used helium (99.999\%) as a carrier gas, which was maintained at a constant flow rate of $1.2 \mathrm{~mL} / \mathrm{min}$. GC temperature changes are as follows: $0-1 \mathrm{~min}, 60^{\circ} \mathrm{C} ; 1-14 \mathrm{~min}, 60-320^{\circ} \mathrm{C}$; and $14-19 \mathrm{~min}$, $320^{\circ} \mathrm{C}$. The electron energy is $70 \mathrm{eV}$, and the MS data were obtained in full scan mode with a mass range of $50-500 \mathrm{~m} / \mathrm{z}$ and a time range of 3.5-19 min. The QC was randomized by sequence, and a QC sample was injected between every 8 
actual sample to check the peak intensity of the spiked internal standard (1,2- ${ }^{13} \mathrm{C}_{2}$-myristic acid).

\section{Electrophysiological tests}

The rats were anesthetized with isoflurane and were checked for pain reflex. Then the rats were fixed on the dissection table to expose the heart. The injection needle was inserted into the right ventricle, to infuse $30-\mathrm{mL}$ icy artificial cerebrospinal fluid (aCSF). And after the perfusion, the brain was decapitated immediately and was placed in ice-cold aCSF for $2 \mathrm{~min}$. aCSF composition was $125 \mathrm{mM} \mathrm{NaCl}, 3.25 \mathrm{mM} \mathrm{KCl}$, $1.25 \mathrm{mM} \mathrm{NaH}_{2} \mathrm{PO}_{4}, 25 \mathrm{mM} \mathrm{NaHCO} 3,1 \mathrm{mM} \mathrm{MgCl} 2 \cdot 6 \mathrm{H}_{2} \mathrm{O}$, $2 \mathrm{mM} \mathrm{CaC}_{12} \cdot 2 \mathrm{H}_{2} \mathrm{O}, 11 \mathrm{mM}$ D-glucose, and $2 \mathrm{mM}$ ascorbate( $\mathrm{pH}$ 7.3-7.4), as previously reported (Wang et al. 2012, 2017). A total of 380- $\mu \mathrm{m}$ hippocampal slices were cut with Vibration slicer (Leica VT1000S), and the tissue was incubated in aCSF which was bubbled with air mixture of 95\% oxygen and 5\% carbon dioxide (Shi et al. 2018). Longterm potentiation (LTP) was assessed by recording field excitatory postsynaptic potentials in the hippocampus.

The whole perfusion system in vitro was continuously perfused with normal aCSF with $95 \%$ oxygen and $5 \%$ carbon dioxide. The temperature of cerebrospinal fluid was maintained at $24{ }^{\circ} \mathrm{C}$ and the flow rate was about $2-3 \mathrm{~mL} / \mathrm{min}$. A borate glass microelectrode filled with aCSF was used to record the evoked excitatory postsynaptic potentials. The concentric bipolar electrode (Bowdoin, ME 04287, USA) was placed in the Schaffer collateral branch to deliver presynaptic stimulation. The glass microelectrode was placed in the stratum radiatum of the CA1 area. The fEPSP corresponding to $30 \%-40 \%$ of the maximum amplitude response is tested. The stimulation electrode was used to deliver the test stimulus every $30 \mathrm{~s}$, with each stimulus lasted $100 \mu \mathrm{s}$. After recording the stable baseline for at least $10 \mathrm{~min}$, the high frequency stimulus program (HFS protocol consisting of 3 bursts of $100 \mathrm{~Hz}, 1 \mathrm{~s}$ pulses at $20 \mathrm{~s}$ intervals) was applied, and LTP was induced. LTP was recorded with the same baseline stimulation intensity (half of maximum stimulation) and recorded for at least $60 \mathrm{~min}$. The slope of the induced fEPSP was measured and normalized to an average baseline of one minute. The field potential recording was filtered at $2 \mathrm{kHz}$, sampled at $20 \mathrm{kHz}$, and continuously recorded using a MultiClamp 700B amplifier (Axon Instruments, USA), and electrophysiological data was analyzed with Clampfit 10.5 software (Molecular Devices). The group allocations of the rats were blinded to the experimenters.

\section{Statistical analysis}

All data are shown as the mean \pm SEM. Differences in behavioral results were evaluated using Student's $t$-test. All statistical analyses were carried out using SPSS software (version
20.0, SPSS Inc., USA). A $p$ value less than 0.05 ( $p<0.05$ ) was considered statistically significant. GC-MS data (Raw Data Files) of all samples were converted using the ABF converter (Ursell et al. 2014) (http://www.reifycs.com/AbfConverter) into MS-DIAL (v.2.7.2) software program (Furness et al. 2014) to perform peak detection, identification, and alignment. Metabolite identification was performed by comparing the MS spectrum and the Ankle RI index with those of the National Institute of Standards and Technology (NIST), and the metabolites were confirmed only when the EI similarity was higher than $80 \%$. Normalized screening used R language after export (Kaelberer et al. 2018). The data were normalized to $\log$ conversion using MetaboAnalyst (http://www. metaboanalyst.ca/) before a series of multivariate statistical analyses, and the statistical significance of the identified metabolites was assessed using one-way ANOVA and $q$-test. The direct threshold changes of the final metabolite $>1.2$ and $p<0.05$ can be considered as potentially altered metabolites. All statistical analyses were then plotted using Prism 8 (GraphPad 8.00) and MetaboAnalyst 4.0.

\section{Results}

\section{UCMS-induced remarkable depression-like behavior}

Adult male Sprague-Dawley (SD) rats were used to investigate the effects of chronic stress on the metabolic changes in both brain and gut. To determine if UCMS could successfully induce depression-like behaviors, we measured behavior changes, body weight, and plasma corticosterone (CORT) concentration of the rats after the UCMS training. Sucrose preference test, forced swim test (FST), and open field test (OFT) were used to test UCMSinduced depression-like behaviors. The results showed that UCMS significantly decreased the sucrose consumption in the sucrose preference test (SPT) $(p<0.01$, compared with UCMS group; $p<0.01$, compared with control group, one-way ANOVA, $n=12$, Fig. 1A). In the forced swim test, the immobility time in the UCMS group was significantly longer than that of the control group at the 5 th week ( $p<0.01$ vs. control, one-way ANOVA, $n=12$, Fig. 1B). At the same time, the results of open field test (OFT) were also analyzed (Fig. 1C, D). Rats exposed to UCMS showed less total distance and fewer grooming bouts $(p<0.05)$, indicating that depression was successfully induced. In addition, we also monitored body weight and plasma corticosterone (CORT) over the UCMS procedure. Our results showed that UCMS significantly decreased body weight and increased the CORT, which is consistent with our previous reports (Fig. 1E, F) (Gu et al. $2018 \mathrm{a}, \mathrm{b})$. These results indicate that UCMS caused depression-like behaviors. 
Fig. 1 Behavior tests and physiological changes after UCMS. A Scores for sucrose preference test; $\mathbf{B}$ forced swim test; $\mathbf{C}$ open field test (distance); D open field test (grooming bouts). Sucrose preference test represents sugar water preference rates, which were calculated with the equation: SPT, sucrose consumption/(sucrose consumption + water consumption); $\mathbf{E}$ body weight changes during the UCMS training; $\mathbf{F}$ corticosterone (cort) levels in the blood $(n=12)$
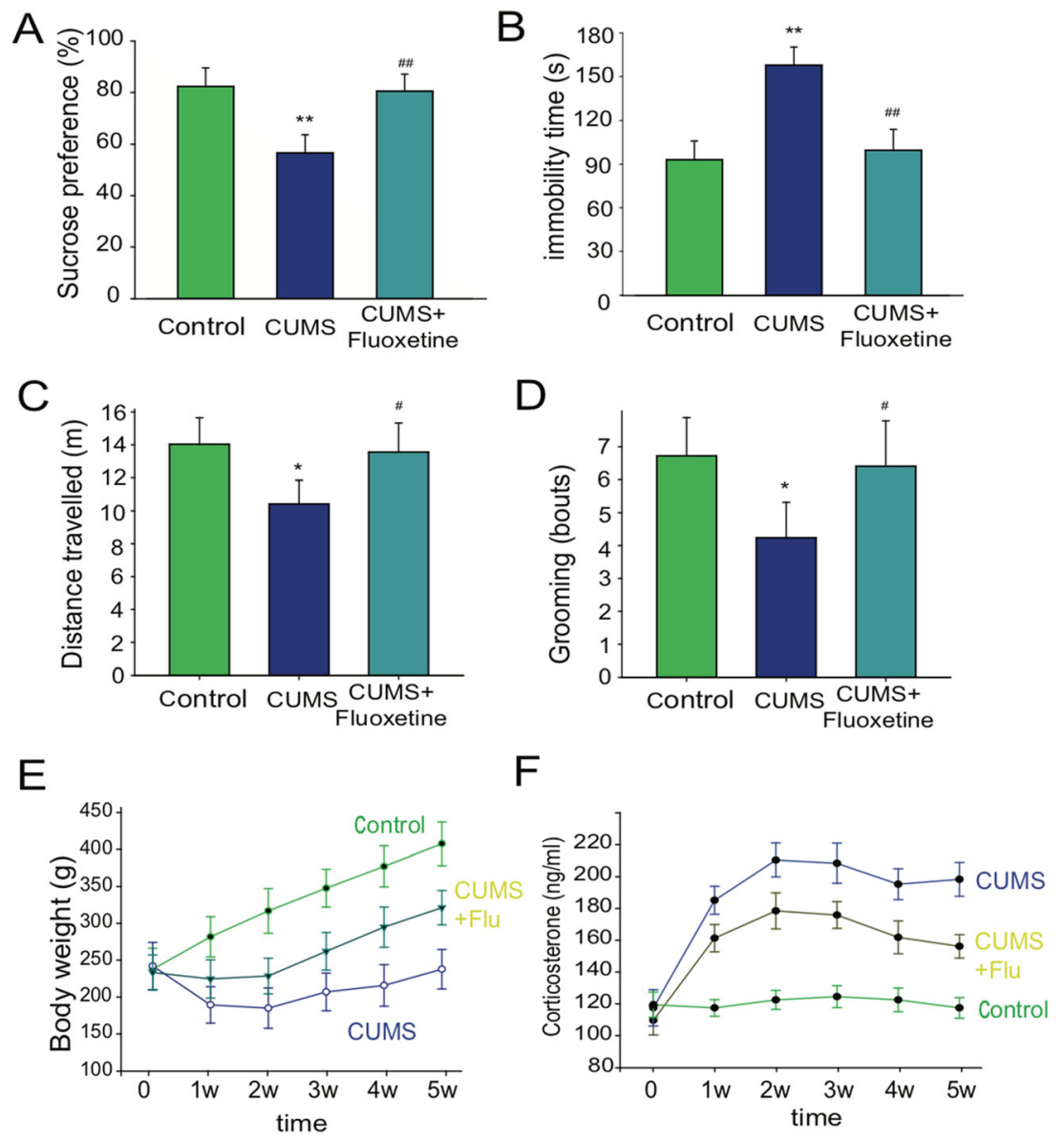

\section{Hippocampus and jejunum metabolomics profile and multivariate data}

We next investigated metabolic changes in UCMS-induced depressive behavior in rats. We tested low-molecular-weight metabolites with GC-MS/MS and expressed as chromatographic peaks, because these small molecules can be easily transported from the gut to brain. The detected metabolites in the hippocampal samples can be called the typical total ion chromatogram (TIC) of the hippocampus (Fig. 2A). In hippocampus samples, 144 endogenous metabolites were identified, including amino acids, glucose, amides, pyrimidines, and fatty acids. In jejunum samples, 190 endogenous metabolites were identified, including amino acids, amino alcohol derivatives, fatty acid, and pyrimidines. In addition, there were 4 metabolites which increased significantly in both hippocampus and jejunum.

\section{Metabolomics profile and multivariate data analysis}

System stability and reproducibility analysis in both hippocampus and jejunum samples were performed based on GCMS to obtain biochemical information on metabolites. The multivariate analysis method of principal component analysis
(PCA) was done to investigate the similarities and differences among the groups. The PCA score scatter plot (Fig. 3) demonstrated significant difference among UCMS group and control group, indicating that UCMS significantly decreased some endogenous metabolite levels in the hippocampus $(p<$ $0.05, t$-test, $n=8$, Fig. $3 \mathrm{~A}$ ), and these metabolite similarly changed in the jejunum of the UCMS rats ( $p<0.05, t$-test, $n=8$, Fig. 3B). To evaluate system stability and reproducibility, PCA analysis was used to process data matrix of quality control (QC) samples. The PCA score plots of the hippocampus and jejunum samples showed that QC samples were clustered, verifying the stability in the GC-MS system throughout the analysis (Supplemental Fig. S1). In addition, the relative standard deviations (RSDs) of the peak height of $1,2-{ }^{13} \mathrm{C}_{2}-$ myristic acid was $13.64 \%$ for the hippocampus and $7.58 \%$ for the jejunum, indicating that the analytical conditions and sample process exhibited good repeatability and stability for the metabolomic study.

There were 48 potential biomarkers in the hippocampus and in the jejunum that significantly changed by UCMS (Table 1), based on one-way ANOVA and $q$-test $(p<0.05)$, and there was a fold change $>1.2$ between the control and UCMS groups. The details of the altered metabolites in the hippocampus and jejunum samples are presented in Table 1. 


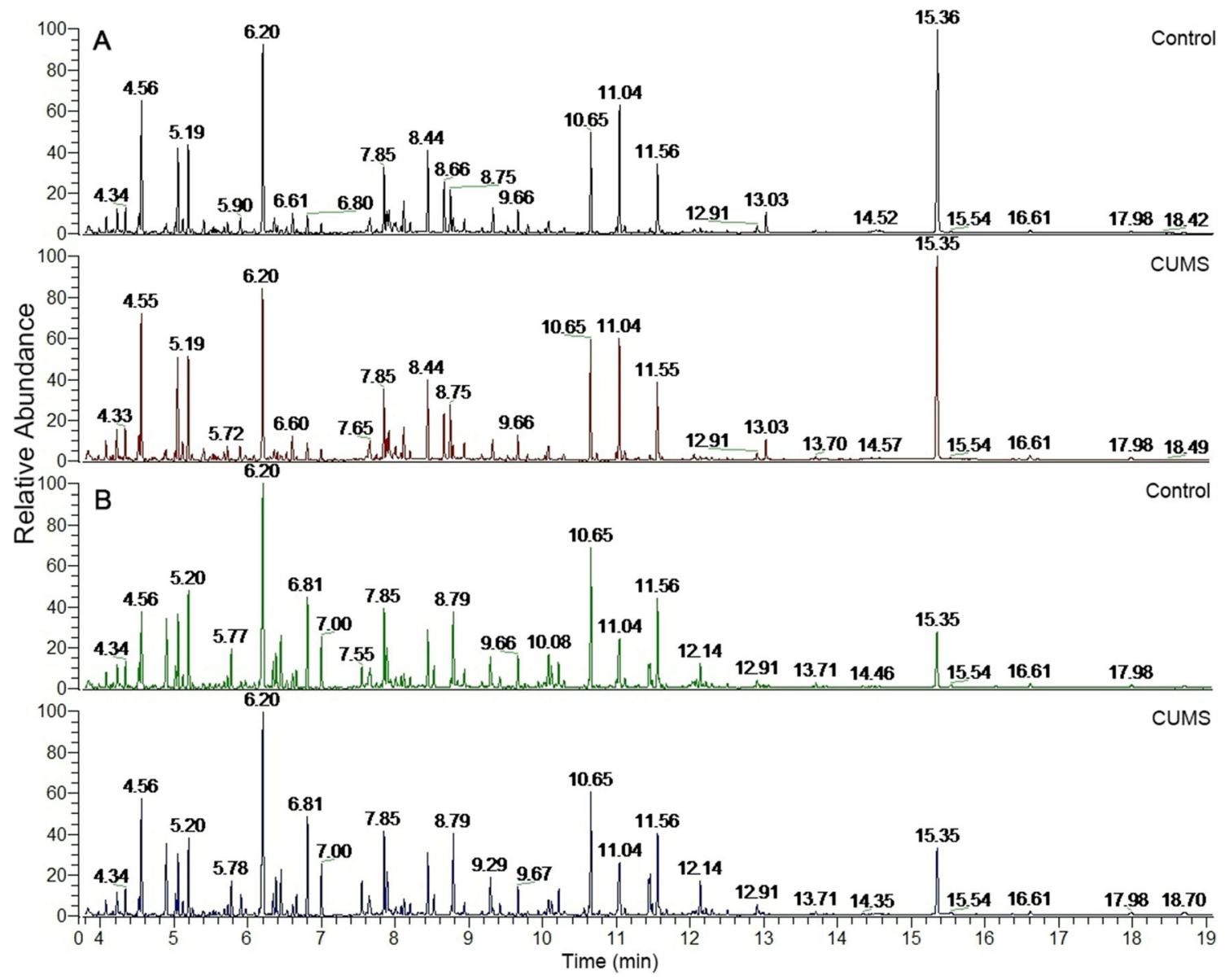

Fig. 2 GC-MS total ion chromatograms (TIC) of hippocampus samples and jejunum samples. A The detected metabolites in the hippocampal samples can be called the typical total ion chromatogram (TIC) of the hippocampus $(n=8)$. In hippocampus samples, 144 endogenous metabolites were identified as changed significantly, including amino acids,

To illustrate the altered metabolites and the content difference among the three groups, metabolic heatmaps of hippocampus and jejunum were also plotted (Fig. 4A, B). It is worth noting glucose, amides, pyrimidines, and fatty acids. B In jejunum samples, 190 endogenous metabolites were identified as changed significantly, including amino acids, amino alcohol derivatives, fatty acid, and pyrimidines

there are more than 4 metabolites that changed more than 1.4folds by UCMS both in the hippocampus and jejunum (Table 1).
Fig. 3 PCA scores scatter plot of hippocampus and jejunum metabolites. A Principal component analysis (PCA) score scatter plot demonstrated a good separation among UCMS group and control, indicating that UCMS significantly decreased some endogenous metabolites levels in hippocampus $(n=8)$. B PCA scatter plot of the metabolites changed in the jejunum of the UCMS rats $(n=8)$
A

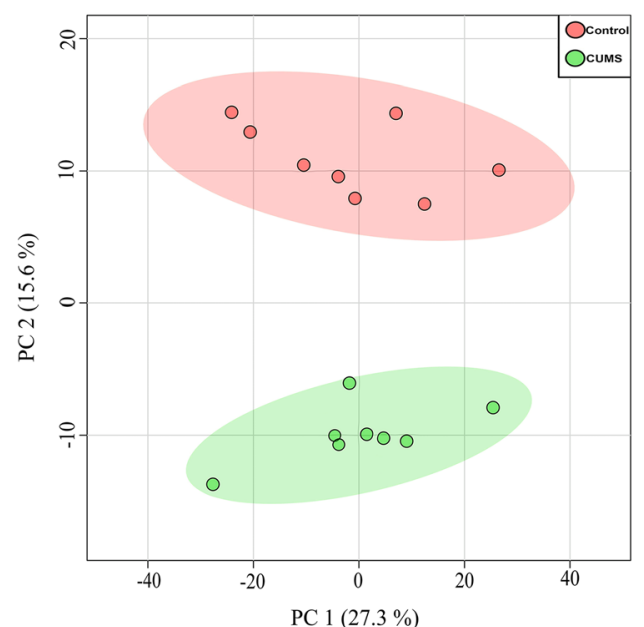

B Scores Plot

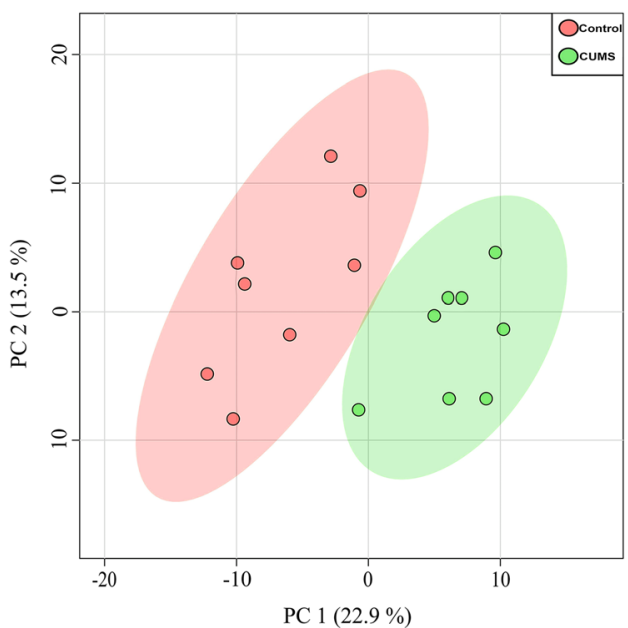


Table 1 Potential biomarkers of the hippocampus and jejunum

\begin{tabular}{|c|c|c|c|c|c|c|}
\hline No. & $\begin{array}{l}\text { Retention } \\
\text { time (min) }\end{array}$ & Metabolites & $\begin{array}{l}\text { Human } \\
\text { Metabolome } \\
\text { Database }\end{array}$ & $\begin{array}{l}\text { Kyoto Encyclopedia } \\
\text { of Genes and Genomes }\end{array}$ & Sites & $\begin{array}{l}\text { Fold change } \\
\text { UCMS vs. control }\end{array}$ \\
\hline 1 & 4.3 & L-arginine & HMDB0000517 & $\mathrm{C} 00062$ & HIP & $0.65085^{* *}$ \\
\hline 2 & 5.77 & L-valine & HMDB0000883 & $\mathrm{C} 00183$ & HIP & $0.8308^{* *}$ \\
\hline 3 & 6 & Benzoic acid & HMDB0001870 & $\mathrm{C} 00180$ & HIP & $0.81405^{* *}$ \\
\hline 4 & 6.34 & L-isoleucine & HMDB0000172 & $\mathrm{C} 00407$ & HIP & $0.77481^{* *}$ \\
\hline 5 & 6.36 & Gamma-aminobutyric acid & HMDB0000112 & $\mathrm{C} 00334$ & HIP & $0.51652^{* *}$ \\
\hline 6 & 6.38 & L-proline & HMDB0000162 & $\mathrm{C} 00148$ & HIP & $0.79225^{* *}$ \\
\hline 7 & 6.45 & Glycine & HMDB0000123 & $\mathrm{C} 00037$ & HIP & $1.4292^{* *}$ \\
\hline 8 & 7.21 & L-aspartic acid & HMDB0000191 & C00049 & HIP & $0.57805^{* *}$ \\
\hline 9 & 8.05 & Threonic acid & HMDB0000943 & $\mathrm{C} 01620$ & HIP & $0.77119^{* *}$ \\
\hline 10 & 8.52 & L-phenylalanine & HMDB0000159 & C00079 & HIP & $0.81452^{*}$ \\
\hline 11 & 9.09 & D-xylitol & HMDB0002917 & C00379 & HIP & $0.78283^{*}$ \\
\hline 12 & 9.29 & Glycerol-1-phosphate & NA & NA & HIP & $0.68143^{* *}$ \\
\hline 13 & 9.32 & p-hydroxyphenylglycine & NA & NA & HIP & $0.72979^{*}$ \\
\hline 14 & 9.42 & O-phosphoethanolamine & HMDB0000224 & $\mathrm{C} 00346$ & HIP & $1.4816^{* *}$ \\
\hline 15 & 9.71 & Phosphoserine & HMDB0000272 & $\mathrm{C} 01005$ & HIP & $0.65319^{*}$ \\
\hline 16 & 9.8 & Dehydroascorbic acid & HMDB0001264 & $\mathrm{C} 00425$ & HIP & $0.69461^{* *}$ \\
\hline 17 & 11.06 & Conduritol B epoxide & NA & NA & HIP & $0.62354^{* *}$ \\
\hline 18 & 11.09 & 2-Hydroxycinnamic acid & HMDB0002641 & $\mathrm{C} 01772$ & HIP & $1.2151^{*}$ \\
\hline 19 & 11.18 & D-Ribulose 5-phosphate & HMDB0000618 & C00199 & HIP & $0.63592^{* *}$ \\
\hline 20 & 11.45 & Oleic acid & HMDB0000207 & $\mathrm{C} 00712$ & HIP & $0.81012^{*}$ \\
\hline 21 & 12.56 & Uridine & HMDB0000296 & C00299 & HIP & $1.2632^{*}$ \\
\hline 22 & 13.59 & Beta-gentiobiose & NA & NA & HIP & $0.31345^{* *}$ \\
\hline 23 & 5.03 & Hydroxylamine & HMDB0003338 & C00192 & HIP and jejunum & $0.78643^{* *}$ and $0.73523^{*}$ \\
\hline 24 & 9.32 & L-glutamine & HMDB0000641 & C00064 & HIP and jejunum & $0.72211^{*}$ and $0.76973^{*}$ \\
\hline 25 & 9.54 & 3-Phosphoglyceric acid & HMDB0000807 & $\mathrm{C} 00597$ & HIP and Jejunum & $0.73102^{* *}$ and $2.2846^{* *}$ \\
\hline 26 & 9.95 & L-tyrosine & HMDB0000158 & C00082 & HIP and jejunum & $0.62828^{* *}$ and $0.7132^{*}$ \\
\hline 27 & 5.49 & Methanolphosphate & NA & NA & Jejunum & $0.38284^{* *}$ \\
\hline 28 & 5.86 & 2-amino-2-methyl-1-3-propanediol & NA & NA & Jejunum & $0.76746^{* *}$ \\
\hline 29 & 5.91 & Urea & HMDB0000294 & C00086 & Jejunum & $4.0981^{* *}$ \\
\hline 30 & 6.03 & Dihydroxyacetone & HMDB0001882 & C00184 & Jejunum & $0.28812^{* *}$ \\
\hline 31 & 6.16 & Ethanolamine & HMDB0000149 & C00189 & Jejunum & $0.61592^{* *}$ \\
\hline 32 & 6.46 & L-carnitine & HMDB0000062 & C00318 & Jejunum & $2.6126^{* *}$ \\
\hline 33 & 6.61 & Iminodiacetic acid & HMDB0011753 & C19911 & Jejunum & $0.76514^{*}$ \\
\hline 34 & 7.09 & Thymine & HMDB0000262 & $\mathrm{C} 00178$ & Jejunum & $0.5547^{* *}$ \\
\hline 35 & 7.26 & Beta-alanine & HMDB0000056 & C00099 & Jejunum & $0.59132^{* *}$ \\
\hline 36 & 8.53 & Anabasine & HMDB0004350 & $\mathrm{C} 06180$ & Jejunum & $0.56955^{*}$ \\
\hline 37 & 8.85 & Pectin & HMDB0003402 & C08348 & Jejunum & $0.33909^{* *}$ \\
\hline 38 & 9.15 & 6-Deoxy-D-glucose & NA & NA & Jejunum & $0.7039^{* *}$ \\
\hline 39 & 9.99 & D-tagatose & HMDB0003418 & $\mathrm{C} 00795$ & Jejunum & $0.25136^{* *}$ \\
\hline 40 & 10.31 & Ascorbic acid & HMDB0000044 & $\mathrm{C} 00072$ & Jejunum & $3.5443^{* *}$ \\
\hline 41 & 10.62 & Gluconic acid & HMDB0000625 & $\mathrm{C} 00257$ & Jejunum & $0.24617^{* *}$ \\
\hline 42 & 11.53 & 5-Hydroxyindoleacetic acid & HMDB0000763 & $\mathrm{C} 05635$ & Jejunum & $1.41^{*}$ \\
\hline 43 & 12.39 & Glucoheptose & NA & NA & Jejunum & $0.43798^{* *}$ \\
\hline 44 & 13 & MG (16:0/0:0/0:0) & HMDB0011564 & NA & Jejunum & $0.70871^{* *}$ \\
\hline 45 & 13.27 & Adenosine & HMDB0000050 & $\mathrm{C} 00212$ & Jejunum & $0.60706^{* *}$ \\
\hline 46 & 13.38 & Sucrose & HMDB0000258 & C00089 & Jejunum & $0.3178^{* *}$ \\
\hline 47 & 13.71 & Glycerol 1-octadecanoate & HMDB0031075 & NA & Jejunum & $0.72799^{*}$ \\
\hline
\end{tabular}


Table 1 (continued)

\begin{tabular}{llllll}
\hline No. & $\begin{array}{l}\text { Retention } \\
\text { time (min) }\end{array}$ & Metabolites & $\begin{array}{l}\text { Human } \\
\text { Metabolome } \\
\text { Database }\end{array}$ & $\begin{array}{l}\text { Kyoto Encyclopedia } \\
\text { of Genes and Genomes }\end{array}$ & $\begin{array}{l}\text { Sites } \\
\text { UCMS vs. control }\end{array}$ \\
\hline 48 & 14.55 & 3'-AMP & HMDB0003540 & C01367 change \\
\hline
\end{tabular}

Note: ${ }^{*} p<0.05,{ }^{* *} p<0.01$. KEGG genome Kyoto Encyclopedia of Genes and Genomes

\section{Trends in common differential metabolites in the hippocampus and jejunum}

The quantification of common metabolites in the hippocampus and jejunum samples in UCMS-induced depression-like behavior in rats were analyzed (Fig. 5). Compared with those in the control group, the concentrations of many substances changed significantly (Table 1 ), for example, $\beta$-gentiobiose decreased to 0.31345 times in the UCMS group compared to normal control. Some substances increased, for example, Lcarnitine increased to 2.6 times. However, the changes are different in the hippocampus and jejunum, the reason might be that the metabolites might be absorbed and transported in different format to the brain. There are four substances - (L-
Fig. 4 Heatmap of identified differential metabolites with hippocampus (A) and jejunum (B) metabolomics profile. Each cell in the heatmap represents the fold change of a particular metabolite. C A Venn diagram generated based on the proportion of the changed substances $(n=8)$
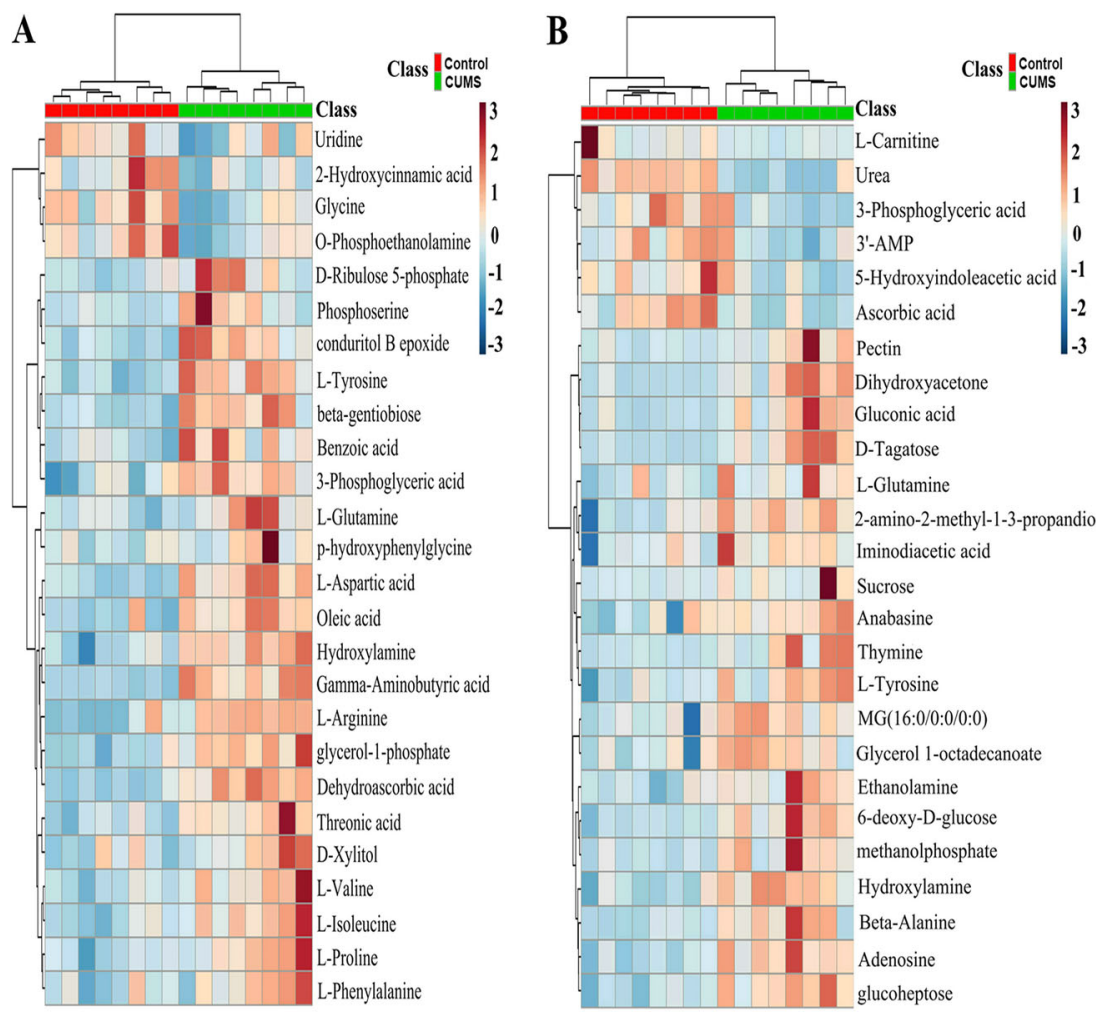

C Hippocampus

Jejunum

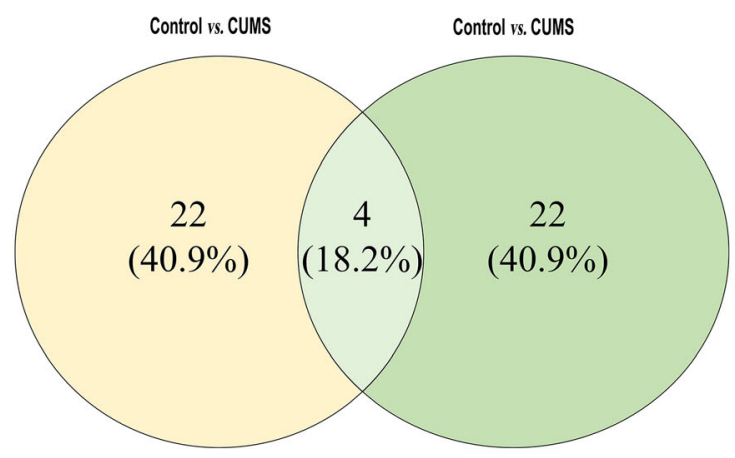


Fig. 5 Scatter plots of significantly changed metabolites normalized peak intensity in rat hippocampus (A) and jejunum samples (B). The $\mathrm{X}$-axis shows the specific metabolite's normalized peak intensity, and each scatter represents a corresponding sample of the rat. orepresents hippocampus samples of control,

$\Delta$ represents hippocampus samples of UCMS, orepresents jejunum samples of control,

$\Delta$ represents jejunum samples of UCMS, the scatter plots show the mean and SD of the metabolites. ${ }^{*} p<0.05, * * p<0.01$, vs. control $(n$ $=8)$
A
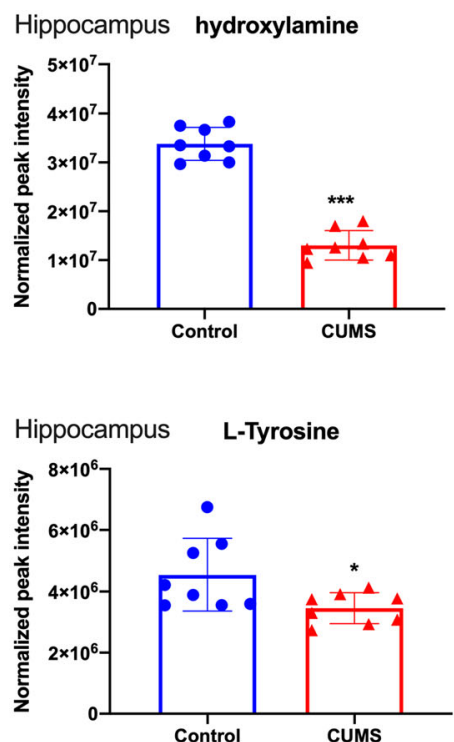

B
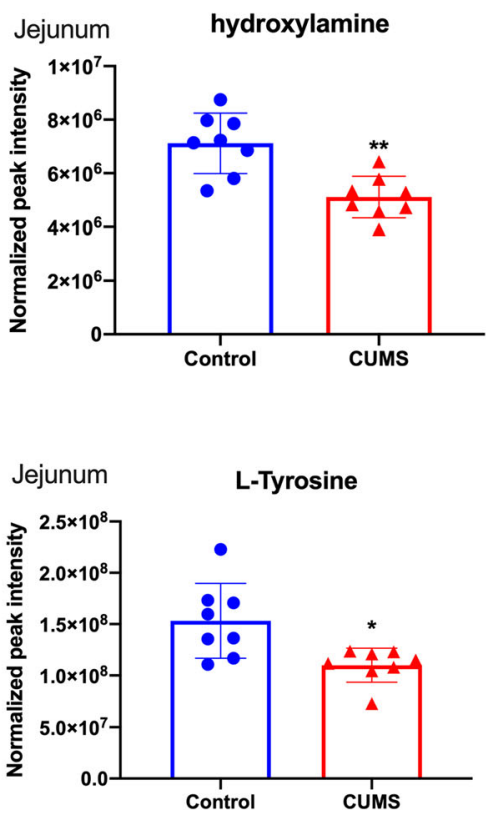
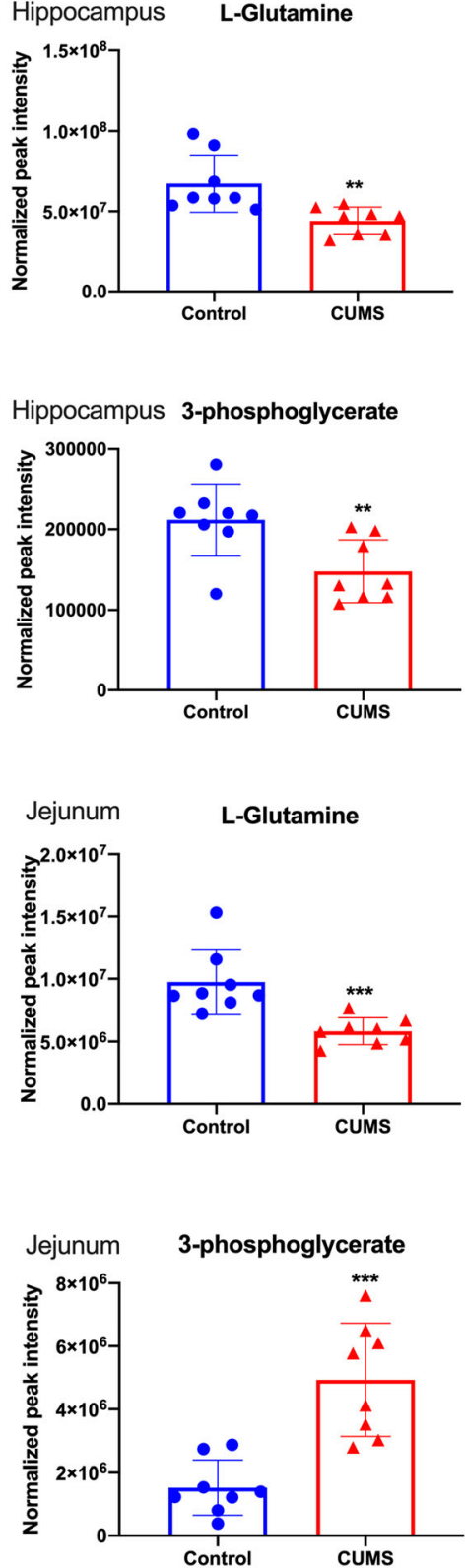

glutamine, L-tyrosine, 3-phosphoglyceric acid, hydroxylamine) - that showed a significant change in both the hippocampus and jejunum. This indicates that metabolism of the hippocampus and jejunum were similarly disturbed in UCMS-induced depression-like behavior in rats.

In order to verify the correlation between the jejunum and hippocampus, the metabolite profile of serum samples was also studied with GC-MS. A total of 101 endogenous metabolites were identified in serum samples. After the data was normalized, QC was used to test the stability of the samples (Fig. S2a), and the correlation between the groups was analyzed with principal component analysis. The normal group and the model group were significantly different (Fig. S2b). The metabolite changes were selected based on a change $>1.2$ between the normal group and UCMS model group (Fig. S3). Consistently, there were 4 metabolites that can be identified; glutamine and L-tyrosine have metabolic changes in peripheral serum that are slightly different from those in the hippocampus and jejunum (Fig. S4).

\section{Neural plasticity changes in the hippocampus}

We recorded LTP in CA1 region of the hippocampal slices of UCMS group rats and control group rats. LTP was successfully inducted and recorded (Fig. 6A) in normal artificial cerebrospinal fluid (aCSF). LTP was triggered by three highfrequency stimuli in both control group and in the UCMS group but was significantly different (calculation was done 
Fig. 6 LTP results from the hippocampus. A LTP decreases in the hippocampus from UCMS mice $(n=4)$. B The record of the experiment operation. $\mathbf{C}$ The statistical analysis of the fEPSPs (field excitatory postsynaptic potentials). Unpaired $t$-test revealed that LTP was significantly decreased in hippocampal slices of UCMS rats. Data are presented as the mean \pm SEM. $* * * p<0.01,(n=$ 4)
A

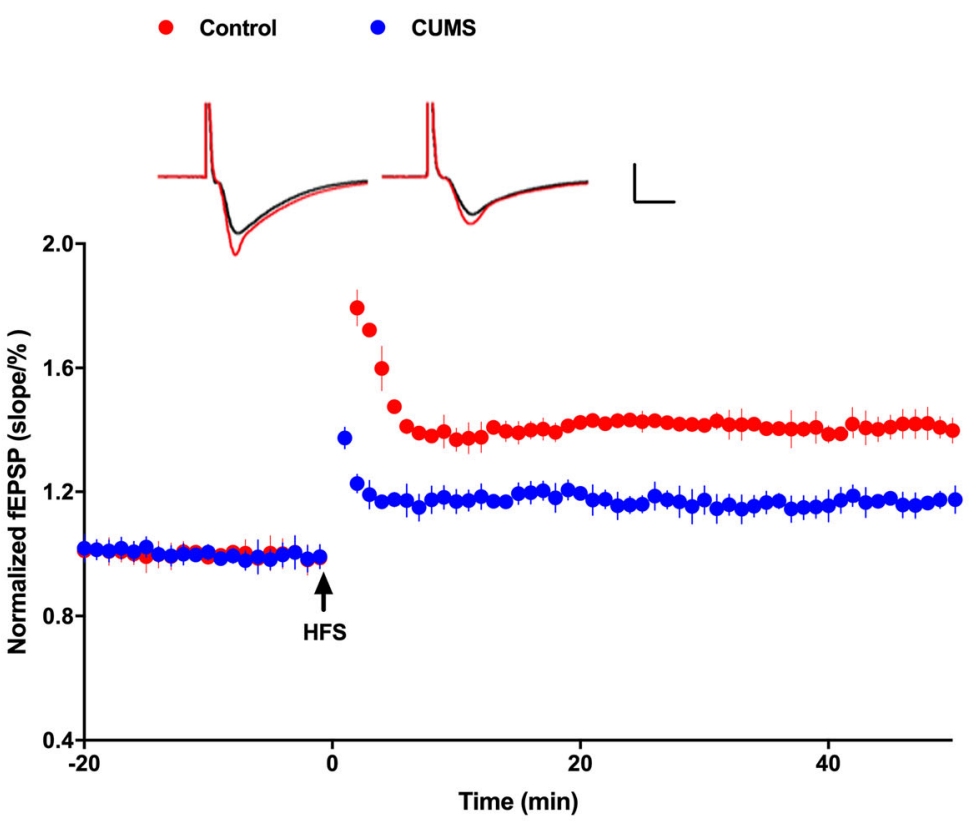

B

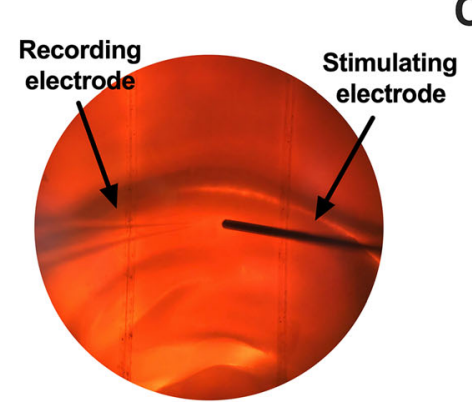

C

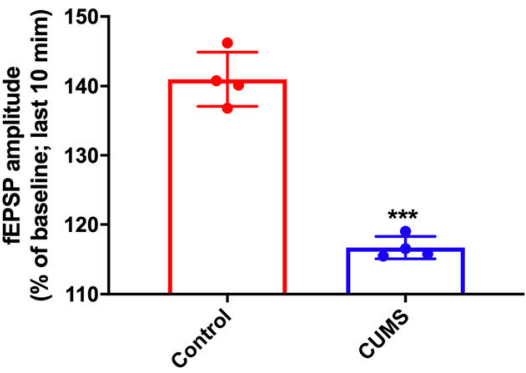

in 4 rats, and averaged amplitude of 10 EPSPs at 10-15 min was used for one animal) (Fig. 6B-C, $* p<0.01, n=4$ ).

\section{Discussion}

In this study, we performed a comprehensive analysis of metabolic changes in brain-gut axis of rats with UCMS-induced depressionlike behavior. Our data show that UCMS-induced depression-like behavior was accompanied with many metabolic changes that similarly happened in both the hippocampus and intestine, such as hydroxylamine, L-glutamine, L-tyrosine, and 3phosphoglyceric acid. These similar metabolite changes in the jejunum and hippocampus confirm that the brain-gut axis is closely related to the pathogenesis of depression-like behavior.

These metabolite changes may be related to microbiota, which has emerged as a key player in control of the brain-gut axis, especially during stressful conditions provoked by real or perceived homeostatic challenge (Hashimoto et al. 2016; Jianguo et al. 2019). Many studies about gut-brain-gut signaling pathways pointed to metabolic changes, such as short chain fatty acids. Metabolites can serve as key messengers for the two-way communication system between the gut and the brain (Benech et al. 2021; Qiao et al. 2014). However, what molecular metabolite plays an important role in the process remains unknown (Kim et al. 2019a). The connection between the metabolic state of the intestines and the central nervous system may be a key to uncover the pathogenesis of depression. As far as we know, this is the first study to investigate the common metabolites affected by brain-gut interaction in depression-like behavior in rats. Various amino acids have been tested in the hippocampus and jejunum of UCMS rats, including proline, arginine, valine $\beta$-alanine, isoleucine, and gamma-aminobutyric acid. Out of the hundreds of metabolites detected in the hippocampus and jejunum samples, 26 metabolites levels were substantially changed and recommended as potential biomarkers for depression (Table 1 and Fig. 7). Using MetaboAnalyst 4.0, Kyoto Encyclopedia of Genes and Genomes (KEGG) (http://www. kegg.jp/), and Human Metabolome Database (HMDB) (http:// www.hmdb.ca/), we conducted metabolic pathway analysis based on the distribution of differential metabolites, and a network of pathways of brain-gut axis metabolism in rats with UCMS-induced depression-like behavior was built with Pathway Builder Tool 2.0 (Fig. 8). 
Fig. 7 Schematic diagram of metabolite distribution.

Metabolites derived from the amino acid difference in the hippocampus. Metabolites derived from the different metabolites of sugar in the jejunum. Different metabolites coexisting in the hippocampus and jejunum
Fig. 8 The pathways closely associated with the depression effects. Bold blue metabolites were potential biomarkers of the hippocampus from depression model; bold black metabolites were potential biomarkers of the jejunum from depression model. Bold red metabolites were potential biomarkers of the hippocampus and jejunum from depression model
Benzoic acid $\circ$

Oleic acid

o Glycine

Threonic acid $\bullet$ L-Arginine

D-Xylitol $\bullet$ L-Proline

Uridine ○ L-Aspartic acid

Phosphoserine o $\bigcirc \mathrm{L}$-Isoleucine

Hippocampus Beta-gentiobiose - L-Phenylalanine

D-Ribulose 5-phosphate 0 Gamma-Aminobutyric acid

Conduritol B epoxide - P-hydroxyphenylglycine

Glycerol-1-phosphate $0 \circ$ O-Phosphoethanolamine Dehydroascorbic acid

\section{3-Phosphoglyceric acid \\ L-Glutamine \\ L-Tyrosine \\ Hydroxylamine}

\section{3-Phosphoglyceric acid} L-Glutamine Hydroxylamine
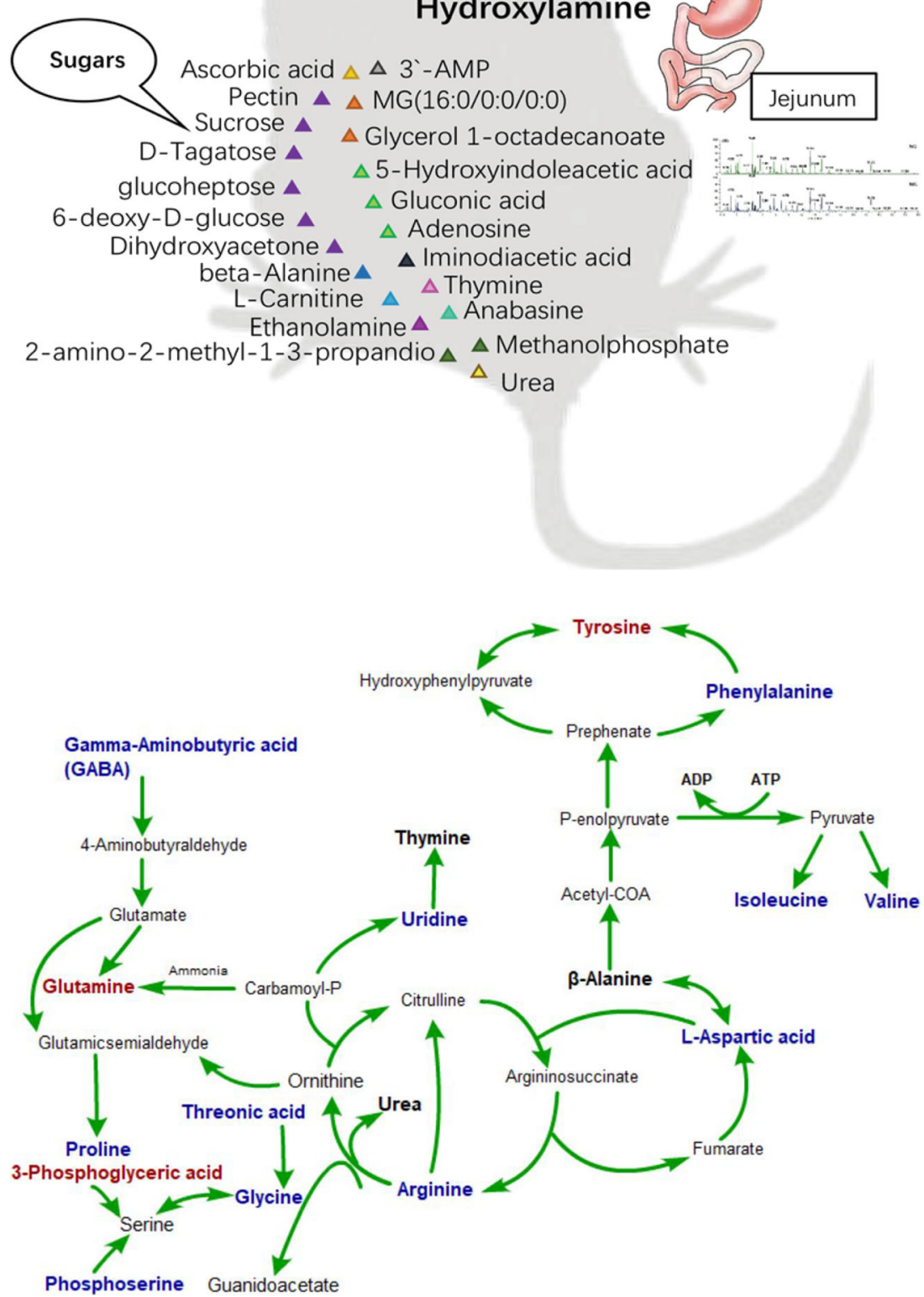
Monoaminergic neurotransmitters have proven to play a key role in the emotion-related disorders, and the first-line of treatments for these diseases are still targeting these neurotransmitters (Gu et al. 2016, 2018a, 2019, Liang et al., 2021, $\mathrm{Xu}$ et al., 2020). In our previous paper, we hypothesized that three monoamines might work as three substrates for the three primary basic emotions: DA-reward-joy, NE- stress (fear and anger), and serotonin-punishment (sad) (Wang et al. 2020, Gu et al. 2019). For example, DA is a catecholamine, which plays an important role in the brain reward system (Wang et al. 2018). Anhedonia, a characteristic feature of major depressive disorder, is often caused by the dysfunction of the reward system in the brain (Burlina et al. 2000). Tyrosine derived from liver phenylalanine (Phe) is a precursor of catecholamines (Ney et al. 2017; Huang et al. 2018), including NE, adrenaline (Ad), and DA. Lack of tyrosine not only leads to insufficient DA synthesis but also weakens the connection between striatum and frontal lobe region, which can induce cognitive impairment (Aleksidze and Blomstrand 1968; Lonart and Johnson 1995). These findings are consistent with the results of our study. Supplementation of tyrosine to individuals with phenylketonuria (PKU) increases the metabolic concentrations of DA and serotonin in cerebrospinal fluid with supplemental probiotics (Gross 1985; Kalmar et al. 2014), suggesting that tyrosine uptake may affect the synthesis of neurotransmitters. Depressive patients often have intestinal problems such as intestinal inflammation (Diamond 1983), and intestinal damage will inevitably impair tyrosine intake. This again proves the correlation between depression and intestinal damage.

Nitric oxide (NO) derived from hydroxylamine and Snitroso-L-cysteine (NO-CYS) can evoke release of hippocampal NE (Morikawa et al. 2007). Being a product of cell metabolism, hydroxylamine, however, can be toxic when it reaches certain level. In brain tissue, it strongly stimulates the oxidation of succinic acid in neurons (Kim et al. 2019b), activates guanylate cyclase in the granule part of cerebral cortex, and inhibits the enzyme activity in glial cells. Hydroxylamine and its derivatives can be neuroprotective and prevent convulsions by suppressing the GABAtransaminase in many neurodegenerative disease models (Glade and Smith 2015; Sabogal-Guáqueta et al. 2018). Normal concentrations of hydroxylamine are associated with the maintenance of normal neural responses in the brain. The hydroxylamine class has also been shown to relax nonvascular smooth muscle at an effective concentration (Edogawa et al. 2020), which can explain the frequent co-occurrence of severe depression (MDD) and irritable bowel syndrome (IBS), as MDD is associated with decreased level of hydroxylamine in intestines.

3-Phosphoglyceric acid is a precursor for serine, which is an endogenous ligand of NMDA-R. Serine concentration in mouse cerebellum is very low (Fernandes and Gupta 2019); however, no change in serine was found in the hippocampus of UCMS rats in this study. The feasibility of using the precursor 3-phosphoglyceric acid content in the hippocampus instead of its content for testing is unknown, but it is certain that L-serine can protect neuronal cells against oxidative stress-mediated apoptotic cell death by contributing to intracellular antioxidant GSH synthesis and maintaining the mitochondrial fusion-fission balance (Qian et al. 2019). It can support human cognitive functions, including formation of shortterm memory, consolidation of long-term memory, learning and recalling information, and development of language and communication skills. Human brain uses serine to synthesize phosphatidylserine. Phosphatidylserine is closely related to aging of human brain, biochemical changes in neurotransmission, and structural deterioration (Cajka et al. 2017). In the case of cerebral ischemia, phosphatidylserine deficiency in the hippocampus leads to cognitive impairment (Tsugawa et al. 2015). All in all, this is related to the content of 3phosphoglyceric acid in rats with depression-like behavior in this study, suggesting that there is some damage to neurons in the brain. Increases of proteolytic activity (PA) in the intestine can disrupt the intestinal shield and increase the intestinal stress response. Serine protects the intestine by inhibiting protease-activated receptor 2 (Par-2) (Xi et al. 2014). The increase in the content of 3-phosphoglycerate related to serine synthesis in this study may be a negative feedback regulation of disease in the intestine of the body. It is speculated that in the UCMS model, substances in the hippocampus have corresponding changes in jejunal metabolism.

Emotional and cognitive impairment and altered hippocampal synaptic plasticity are important features of depression (Tsugawa et al. 2015). Synaptic plasticity is known to regulate and support signal transduction between neurons, and synaptic dysfunction leads to a variety of neurological disorders. Exposure to chronic unpredictable mild stress stimuli in rats for a period of time has led to obstruction of information transmission between glial cells and neurons and decrease of LTP induction at the CA3 to CA1 synapses of the hippocampus (Xi et al. 2014; L'Huillier et al. 2019). These changes in structure and plasticity will further impair hippocampusdependent learning and memory functions (L'Huillier et al. 2020). Glutamine is a precursor for glutamate, which is the main excitatory neurotransmitter in the brain, and serves as the neurotransmitter between endocrine cells and neurons (Kong et al. 2018). The LTP of CA3-CA1 in the hippocampal synapse requires glutamate-mediated activation of NMDA receptor production (Gracie et al. 2019). Intervention of the glutamate system may be the underlying antidepressant mechanism of ketamine, which is a non-competitive antagonist of the Nmethyl-D-aspartate (NMDA) type of glutamate receptor (Zamani et al. 2019). In this study, we found that metabolic disorder of glutamine-glutamic acid cycle in brain-gut network is related to the LTP changes. Traditionally treated as a 
nutritious nonessential amino acid, glutamine is now recognized as a key player in activating the mammalian target of rapamycin cell signal transduction in intestinal cells, promoting proliferation and survival of intestinal cells, repairing intestinal mucosa and maintaining intestinal integrity under stress, and protecting the intestinal tract from atrophy and injury (Gracie et al. 2019; Mahar et al. 2021). Changes in glutamine metabolism in the intestine can induce intestinal shielding dysfunction (LaVoie et al. 2005) and regulate the homeostasis (differentiation, proliferation, renewal) and immune response of intestinal epithelium. The decrease of glutamine content in the intestine and brain could explain that psychological disorder is associated with recurrence of intestinal disease (Spies et al. 2020) and that the incidence of depression in patients with intestinal disease is three times higher than that in normal people (Hensel et al. 2019). Additionally, growing evidence suggests that glutamine holds great potential for being a necessary amino acid supplement in the treatment of depression.

\section{Conclusion}

In conclusion, our study suggests that UCMS-induced depression-like behavior in rats displayed inhibited LTP of Schaffer collateral-CA1 synapses in the hippocampus and metabolic alterations of the brain-gut axis. The underlying mechanisms for this association and how these metabolic alterations interact with synaptic plasticity warrant further investigation. This study is expected to shed light on the neurobiology of depression and will guide the development of new treatment for this menacing neuropsychiatric disorder.

Supplementary Information The online version contains supplementary material available at https://doi.org/10.1007/s00213-021-05958-w.

Acknowledgements The paper was supported by a grant from Foundation of Humanities and Arts from the Ministry of Education in China (19YJAZH083).

Author contribution QX, SX, and FW conceived the study; QX and SG collected and analyzed the behavioral experiments and LTP data; XM, GF, XZ, and MJ conducted GC-MS/MS measurements, collected and analyzed the data; FW, EW, and JHH wrote the manuscript; GF, XM, and SX revised the manuscript.

\section{Declarations}

Ethics approval All animal experiments are conducted in accordance with Guide for the Care and Use of Laboratory Animals (the National Research Council of the National Academies, Eighth Edition, 2011 revision) and carried out in accordance with the NIH Guide for the Care and Use of Laboratory Animals (NIH Publication No. 85-23, 1985, revised 1996) and also performed strictly under the institutional guidelines of the Animal Care and Use Committee of Nanjing University of Chinese Medicine (Nanjing, China).
Competing interests The authors declare no competing interests.

Open Access This article is licensed under a Creative Commons Attribution 4.0 International License, which permits use, sharing, adaptation, distribution and reproduction in any medium or format, as long as you give appropriate credit to the original author(s) and the source, provide a link to the Creative Commons licence, and indicate if changes were made. The images or other third party material in this article are included in the article's Creative Commons licence, unless indicated otherwise in a credit line to the material. If material is not included in the article's Creative Commons licence and your intended use is not permitted by statutory regulation or exceeds the permitted use, you will need to obtain permission directly from the copyright holder. To view a copy of this licence, visit http://creativecommons.org/licenses/by/4.0/.

\section{References}

Aleksidze N, Blomstrand C (1968) The influence of hydroxylamine, thiosemicarbazide and gamma-aminobutyric acid upon succinate oxidation by Deiters' nerve cells and neuroglia. Brain Res 11:717719. https://doi.org/10.1016/0006-8993(68)90166-2

Baj A, Moro E, Bistoletti M, Orlandi V, Crema F, Giaroni C (2019) Glutamatergic signaling along the microbiota-Gut-brain axis. Int $\mathrm{J}$ Mol Sci 20(20): 1482

Benech N, Nathalie R, Harry S (2021) Tryptophan metabolites get the gut moving. Cell Host Microbe 29(2):145-147. https://doi.org/10.1016/ j.chom.2021.01.009

Breit S, Kupferberg A, Rogler G, Hasler G (2018) Vagus nerve as modulator of the brain-gut axis in psychiatric and inflammatory disorders. Front Psychiatry 9:44

Burlina AB, Bonafé L, Ferrari V, Suppiej A, Zacchello F, Burlina AP (2000) Measurement of neurotransmitter metabolites in the cerebrospinal fluid of phenylketonuric patients under dietary treatment. J Inherit Metab Dis 23:313-316. https://doi.org/10.1023/A: 1005694122277

Cajka T, Smilowitz JT, Fiehn O (2017) Validating quantitative untargeted lipidomics across nine liquid chromatography-high-resolution mass spectrometry platforms. Anal Chem 89:12360-12368. https://doi. org/10.1021/acs.analchem.7b03404

Cryan JF, O'Riordan KJ, Cowan CSM, Sandhu KV, Bastiaanssen TFS, Boehme M, Codagnone MG (2019) The Microbiota-Gut-Brain Axis. Physiol Rev 99:1877-2013. https://doi.org/10.1152/physrev. 00018.2018

Diamond J (1983) Lack of correlation between cyclic GMP elevation and relaxation of nonvascular smooth muscle by nitroglycerin, nitroprusside, hydroxylamine and sodium azide. J Pharmacol Exp Ther 225:422-426

Duman RS, Aghajanian GK, Sanacora G, Krystal JH (2016) Synaptic plasticity and depression: new insights from stress and rapid-acting antidepressants. Nat Med 22:238-249. https://doi.org/10.1038/nm. 4050

Edogawa S, Edwinson AL, Peters SA, Chikkamenahalli LL, Sundt W, Graves S, Gurunathan SV, Breen-Lyles M, Johnson S, Dyer R, Graham R, Chen J, Kashyap P, Farrugia G, Grover M (2020) Serine proteases as luminal mediators of intestinal barrier dysfunction and symptom severity in IBS. Gut 69:62-73

Fernandes J, Gupta GL (2019) N-acetylcysteine attenuates neuroinflammation associated depressive behavior induced by chronic unpredictable mild stress in rat. Behav Brain Res 364:356-365. https:// doi.org/10.1016/j.bbr.2019.02.025

Furness JB, Callaghan BP, Rivera LR, Cho H-J (2014) The enteric nervous system and gastrointestinal innervation: integrated local and central control. Adv Exp Med Biol 817:39-71 
Glade MJ, Smith K (2015) Phosphatidylserine and the human brain. Nutrition 31:781-786. https://doi.org/10.1016/j.nut.2014.10.014

Gracie DJ, Hamlin PJ, Ford AC (2019) The influence of the brain-gut axis in inflammatory bowel disease and possible implications for treatment. Lancet Gastroenterol Hepatol 4:632-642. https://doi.org/10. 1016/S2468-1253(19)30089-5

Gross P (1985) Biologic activity of hydroxylamine: a review. Crit Rev Toxicol 14:87-99

Gu S, Jing L, Li Y, Huang JH, Wang F (2018a) Stress induced hormone and neuromodulator changes in menopausal depressive rats. Front Psychiatry 9:253

Gu S, Ma Y, Ge K, Nie R, Wu E, Li Y (2018b) Danshen-Honghua ameliorates stress-induced menopausal depression in rats. Neural Plast 2018:6589608

Gu S, Wang F, Cao C, Wu E, Tang YY, Huang JH (2019) An integrative way for studying neural basis of basic emotions with fMRI. Front Neurosci 13:628

Gu S, Liu Y, Liang F, Feng R, Li Y, Liu G, Gao M, Liu W, Wang F, Huang JH (2020) Screening depressive disorders with tree-drawing test. Front Psychol 11:1446

Gu S, Wang W, Wang F, Huang JH (2016) Neuromodulator and emotion biomarker for stress induced mental disorders. Neural Plast 2016: 2609128. https://doi.org/10.1155/2016/2609128

Hashimoto K, Bruno D, Nierenberg J, Marmar CR, Zetterberg H, Blennow K, Pomara N (2016) Abnormality in glutamineglutamate cycle in the cerebrospinal fluid of cognitively intact elderly individuals with major depressive disorder: a 3-year follow-up study. Transl Psychiatry 6:e744. https://doi.org/10.1038/tp.2016.8

Hensel C, Becker M, Düzel S, Demuth I, Norman K, SteinhagenThiessen E, Gallinat J, Lindenberger U, Kühn S (2019) Influence of nutritional tyrosine on cognition and functional connectivity in healthy old humans. Neuroimage 193:139-145. https://doi.org/10. 1016/j.neuroimage.2019.03.005

Huang Y, Shi X, Li Z, Shen Y, Shi X, Wang L, Li G, Yuan Y, Wang J, Zhang Y, Zhao L, Zhang M, Kang Y, Liang Y (2018) Possible association of Firmicutes in the gut microbiota of patients with major depressive disorder. Neuropsychiatr Dis Treat 14:3329-3337

Iqbal J, Adu-Nti F, Wang X, Qiao H, Ma XM (2020) Sex difference in depression: which animal models mimic it. Behav Neurosci 134(3): 248-266. https://doi.org/10.1037/bne0000369

Jianguo L, Xueyang J, Cui W, Changxin W, Xuemei Q (2019) Altered gut metabolome contributes to depression-like behaviors in rats exposed to chronic unpredictable mild stress. Transl Psychiatry 9:40

Kaelberer MM, Buchanan KL, Klein ME, Barth BB, Montoya MM, Shen X, Bohórquez DV (2018) A gut-brain neural circuit for nutrient sensory transduction. Science 361:5236

Kalmar B, Lu C-H, Greensmith L (2014) The role of heat shock proteins in amyotrophic lateral sclerosis: the therapeutic potential of arimoclomol. Pharmacol Ther 141:40-54. https://doi.org/10.1016/ j.pharmthera.2013.08.003

Kim J, Farchione T, Potter A, Chen Q, Temple R (2019a) Esketamine for treatment-resistant depression - first FDA-approved antidepressant in a New class. N Engl J Med 381:1-4

Kim KY, Hwang SK, Park SY, Kim MJ, Jun DY, Kim YH (2019b) 1Serine protects mouse hippocampal neuronal HT22 cells against oxidative stress-mediated mitochondrial damage and apoptotic cell death. Free Radic Biol Med 141:447-460

Kong S, Zhang YH, Zhang W (2018) Regulation of intestinal epithelial cells properties and functions by amino acids. Biomed Res Int 2018: 2819154. https://doi.org/10.1155/2018/2819154

LaVoie MJ, Ostaszewski BL, Weihofen A, Schlossmacher MG, Selkoe DJ (2005) Dopamine covalently modifies and functionally inactivates parkin. Nat Med 11:1214-1221. https://doi.org/10.1038/ $\mathrm{nm} 1314$

L'Huillier C, Jarbeau M, Achamrah N, Belmonte L, Amamou A, Nobis S, Goichon A et al (2019) Glutamine, but not branched-chain amino acids, restores intestinal barrier function during activity-based anorexia. Nutrients 11:1348. https://doi.org/10.3390/nu11061348

L'Huillier C, Jarbeau M, Pingeon F, Bahlouli W, Salameh E, Amamou A, Goichon A et al (2020) Influence of glutamine and branched-chain amino acids supplementation during refeeding in activity-based anorectic mice. Nutrients 12:3510. https://doi.org/10.3390/ nu12113510

Liang F, Xu Q, Jiang M, Feng R, Jiang S, Yuan B et al (2021) Emotion induced monoamine neuromodulator release affects functional neurological disorders. Front Cell Dev Biol 15(9):633048

Liang Y, Yu B, Wang Y, Qiao Z, Cao T, Zhang P (2018) Jejunal long noncoding RNAs are associated with glycemic control via gut-brain axis after bariatric surgery in diabetic mice. Surg Obes Relat Dis 14: 821-832. https://doi.org/10.1016/j.soard.2018.03.006

Lonart G, Johnson KM (1995) Characterization of nitric oxide generatorinduced hippocampal $[3 \mathrm{H}]$ norepinephrine release. II. The role of calcium, reverse norepinephrine transport and cyclic 3',5'-guanosine monophosphate. J Pharmacol Exp Ther 275:14-22

Mahar Rohit, Zeng Huadong, Giacalone Anthony, Ragavan Mukundan, Mareci Thomas H, Merritt Matthew E (2021) Deuterated water imaging of the rat brain following metabolism of $[\mathrm{H}]$ glucose. Magn Reson Med, Online ahead of print, DOI: https://doi.org/10.1111/ajd. 13676

Morikawa A, Hamase K, Inoue T, Konno R, Zaitsu K (2007) Alterations in D-amino acid levels in the brains of mice and rats after the administration of D-amino acids. Amino Acids 32:13-20. https://doi. org/10.1007/s00726-005-0357-8

Ney DM, Murali SG, Stroup BM, Nair N, Sawin EA, Rohr F, Levy HL (2017) Metabolomic changes demonstrate reduced bioavailability of tyrosine and altered metabolism of tryptophan via the kynurenine pathway with ingestion of medical foods in phenylketonuria. Mol Genet Metab 121:96-103. https://doi.org/10.1016/j.ymgme.2017. 04.003

Otte C, Gold SM, Penninx BW, Pariante CM, Etkin A, Fava M, Mohr DC, Schatzberg AF (2016) Major depressive disorder. Nat Rev Dis Primers 2:16065

Putnam KT, Wilcox M, Robertson-Blackmore E, Sharkey K, Bergink V, Munk-Olsen T, Deligiannidis KM, Payne J, Altemus M, Newport J, Apter G, Devouche E, Viktorin A, Magnusson P, Penninx B, Buist A, Bilszta J, O'Hara M, Stuart S et al (2017) Clinical phenotypes of perinatal depression and time of symptom onset: analysis of data from an international consortium. Lancet Psychiatry 4:477-485. https://doi.org/10.1016/S2215-0366(17)30136-0

Qian W, Shan J, Shen C, Yang R, Xie T, Di L (2019) Brain metabolomics reveal the antipyretic effects of Jinxin oral liquid in young rats by using gas chromatography-mass spectrometry. Metabolites 9(1):6. https://doi.org/10.3390/metabo9010006

Qiao H, An S-C, Ren W, Ma X-M (2014) Progressive alterations of hippocampal CA3-CA1 synapses in an animal model of depression. Behav Brain Res 275:191-200. https://doi.org/10.1016/j.bbr.2014. 08.040

Qin XH, Wu Z, Dong JH, Zeng YN, Xiong WC, Liu C, Wang MY et al (2019) Liver soluble epoxide hydrolase regulates behavioral and cellular effects of chronic stress. Cell Rep 29:3223-3234.e6. https://doi.org/10.1016/j.celrep.2019.11.006

Sabogal-Guáqueta AM, Villamil-Ortiz JG, Arias-Londoño JD, CardonaGómez GP (2018) Inverse phosphatidylcholine/ phosphatidylinositol levels as peripheral biomarkers and phosphatidylcholine/lysophosphatidylethanolaminephosphatidylserine as hippocampal indicator of postischemic cognitive impairment in rats. Front Neurosci 12:989

Sharon G, Cruz NJ, Kang DW, Gandal MJ, Wang B, Kim YM, Zink EM et al (2019) Human gut microbiota from autism spectrum disorder promote behavioral symptoms in mice. Cell 177:1600-1618.e17. https://doi.org/10.1016/j.cell.2019.05.004 
Shi H, Zhang X, Weng YL, Lu Z, Liu Y, Lu Z, Li J, Hao P, Zhang Y, Zhang F, Wu Y, Delgado JY, Su Y, Patel MJ, Cao X, Shen B, Huang X, Ming GL, Zhuang X et al (2018) m6A facilitates hippocampus-dependent learning and memory through YTHDF1. Nature 563(7730):249-253. https://doi.org/10.1038/s41586-0180666-1

Spies G, Mall S, Wieler H, Masilela L, Castelon Konkiewitz E, Seedat S (2020) The relationship between potentially traumatic or stressful events, HIV infection and neurocognitive impairment (NCI): a systematic review of observational epidemiological studies. Eur J Psychotraumatol 11:1781432. https://doi.org/10.1080/20008198. 2020.1781432

Strandwitz P (2018) Neurotransmitter modulation by the gut microbiota. Brain Res 1693:128-133. https://doi.org/10.1016/j.brainres.2018. 03.015

Tsugawa H, Cajka T, Kind T, Ma Y, Higgins B, Ikeda K, Kanazawa M, VanderGheynst J, Fiehn O, Arita M (2015) MS-DIAL: dataindependent $\mathrm{MS} / \mathrm{MS}$ deconvolution for comprehensive metabolome analysis. Nat Methods 12:523-526. https://doi.org/10.1038/nmeth. 3393

Ursell LK, Haiser HJ, Van Treuren W, Garg N, Reddivari L, Vanamala J, Dorrestein PC et al (2014) The intestinal metabolome: an intersection between microbiota and host. Gastroenterology 146:1470 1476. https://doi.org/10.1053/j.gastro.2014.03.001

Wang F, Pan F, Shapiro LA, Huang JH (2018) Stress induced neuroplasticity and mental disorders 2018. Neural Plast 2018: 5382537. https://doi.org/10.1155/2018/5382537

Wang F, Wang X, Shapiro LA, Cotrina ML, Liu W, Wang EW, Gu S, Wang W, He X, Nedergaard M, Huang JH (2017) NKCC1 up- regulation contributes to early post-traumatic seizures and increased post-traumatic seizure susceptibility. Brain Struct Funct 222:15431556. https://doi.org/10.1007/s00429-016-1292-z

Wang F, Xu Q, Wang W, Takano T, Nedergaard M (2012) Bergmann glia modulate cerebellar Purkinje cell bistability via Ca2+-dependent K+ uptake. Proc Natl Acad Sci U S A 109:7911-7916

Wang F, Yang J, Pan F, Ho RC, Huang H (2020) Editorial: neurotransmitters and emotions. Front Psychol 11:21

Xi B, Gu H, Baniasadi H, Raftery D (2014) Statistical analysis and modeling of mass spectrometry-based metabolomics data. Methods Mol Biol 1198:333-353

Xu Q, Jiang M, Gu S, Wang F, Yuan B (2020) Early life stress induced DNA methylation of monoamine oxidases leads to depressive-like behavior. Front Cell Dev Biol 8:582247

Zamani M, Alizadeh-Tabari S, Zamani V (2019) Systematic review with meta-analysis: the prevalence of anxiety and depression in patients with irritable bowel syndrome. Aliment Pharmacol Ther 50:132143. https://doi.org/10.1111/apt.15325

Zheng P, Zeng B, Zhou C, Liu M, Fang Z, Xu X, Zeng L, Chen J, Fan S, du X, Zhang X, Yang D, Yang Y, Meng H, Li W, Melgiri ND, Licinio J, Wei H, Xie P (2016) Gut microbiome remodeling induces depressive-like behaviors through a pathway mediated by the host's metabolism. Mol Psychiatry 21:786-796. https://doi.org/10.1038/ mp.2016.44

Publisher's note Springer Nature remains neutral with regard to jurisdictional claims in published maps and institutional affiliations. 